\title{
BMJ
}

\section{Adoption, non-adoption, and abandonment of a personal electronic health record: case study of HealthSpace}

\author{
Trisha Greenhalgh, director, ${ }^{1}$ Susan Hinder, freelance researcher, ${ }^{2}$ Katja Stramer, senior research fellow, ${ }^{3}$ \\ Tanja Bratan, research fellow, ${ }^{3}$ jill Russell, senior lecturer $^{3}$
}

${ }^{1}$ Healthcare Innovation and Policy Unit, Centre for Health Sciences, Barts and the London School of Medicine and Dentistry, London E1 2AT, UK

${ }^{2}$ RAFT Research and Consulting, Downham, Clitheroe BB7 4BN, UK

${ }^{3}$ Division of Medical Education, University College London

Correspondence to: T Greenhalgh p.greenhalgh@qmul.ac.uk

Cite this as: BMJ 2010;341:C5814 doi:10.1136/bmi.c5814

\section{ABSTRACT}

Objective To evaluate the policy making process, implementation by NHS organisations, and patients' and carers' experiences of efforts to introduce an internet accessible personal electronic health record (HealthSpace) in a public sector healthcare system. Design Mixed method, multilevel case study. Setting English National Health Service; the basic HealthSpace technology (available throughout England) and the advanced version (available in a few localities where this option had been introduced) were considered. Main outcome measures National statistics on invitations sent, HealthSpace accounts created, and interviews and ethnographic observation of patients and carers. Data analysis was informed by a socio-technical approach which considered macro and micro influences on both adoption and non-adoption of innovations, and by the principles of critical discourse analysis.

Participants 56 patients and carers (of whom 21 opened a basic HealthSpace account, 20 had diabetes but were not initially using HealthSpace, and 15 used advanced HealthSpace accounts to exchange messages with their general practitioner), 3000 pages of documents (policies, strategies, business plans, minutes of meetings, correspondence), observational field notes, and 160 interviews with policy makers, project managers, and clinical staff.

Results Between 2007 and October 2010, 172950 people opened a basic HealthSpace account. 2913 ( $0.13 \%$ of those invited) opened an advanced account, compared with $5-10 \%$ of the population anticipated in the original business case. Overall, patients perceived HealthSpace as neither useful nor easy to use and its functionality aligned poorly with their expectations and self management practices. Those who used email-style messaging were positive about its benefits, but enthusiasm beyond three early adopter clinicians was low, and fewer than 100 of 30000 patients expressed interest. Policy makers' hopes that "deploying" HealthSpace would lead to empowered patients, personalised care, lower NHS costs, better data quality, and improved health literacy were not realised over the three year evaluation period.

Conclusion Unless personal electronic health records align closely with people's attitudes, self management practices, identified information needs, and the wider care package (including organisational routines and incentive structures for clinicians), the risk that they will be abandoned or not adopted at all is substantial. Conceptualising such records dynamically (as components of a socio-technical network) rather than statically (as containers for data) and employing user centred design techniques might improve their chances of adoption and use. The findings raise questions about how eHealth programmes in England are developed and approved at policy level.

\section{INTRODUCTION}

In Britain as in many other countries, the prevalence of long term conditions is increasing but the budget for providing services is falling in real terms. ${ }^{1}$ Patients' involvement in their care is viewed by some as both inherently desirable (empowering) and potentially cost saving. ${ }^{2}$ The vision for informed, self managing patients as active partners in healthcare represents a major cultural shift from the paternalistic, disease focused models of the past (fig 1). ${ }^{34}$ Personal electronic health records, managed by patients and interfacing with clinician held records, are seen as having a key role in the new care model, by facilitating storage and exchange of information, promoting engagement with self management, and supporting continuity of care, ${ }^{5-9}$ although they raise practical, ethical, legal, clinical, technical, cultural, and financial challenges. ${ }^{610-15}$

Provision of personal electronic health records as an integral part of a wider care package may reduce clinician workload and overall healthcare costs. The US health maintenance organisation Kaiser Permanente, for example, offers an electronic record system that includes "My Health Manager," a customisable portal through which members may access parts of their centrally held record, including laboratory results, drugs, allergies, immunisations, past clinic visits, and key diagnoses; patients can email their physician through a secure connection. ${ }^{1617}$ By mid-2008, 2.4 million of Kaiser's 8.7 million members had registered for My Health Manager, most commonly for repeat prescriptions. Use of the system was associated with up to $10 \%$ fewer visits to the physician and a significant reduction in telephone calls. ${ }^{18}$ A survey of members who were 


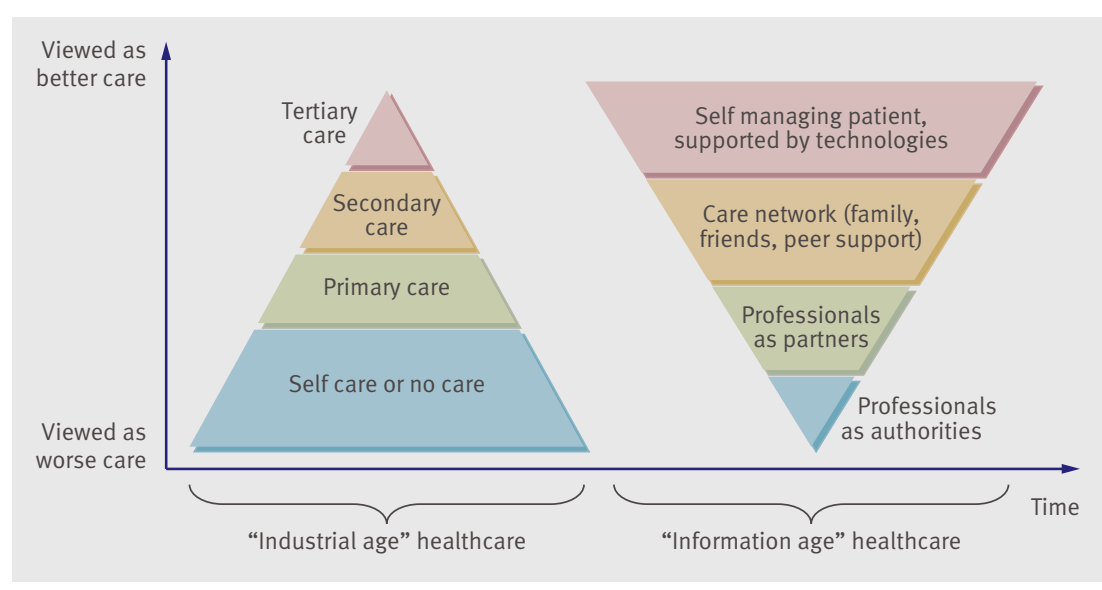

Fig 1| Policy vision of specialist driven care shifting to patient driven care as information age replaces industrial age. Adapted from Ferguson ${ }^{8}$

actively using this technology showed that most perceived it as useful and easy to use. ${ }^{16}$

Other studies, however, have shown slow uptake of using personal electronic health records by both patients and clinicians, low levels of awareness, and unrealistic expectations. ${ }^{1019}$ The material properties and functionality of the technology seem to be important determinants of uptake and use. ${ }^{1320}$ Some doctors have expressed concern that "untethered" personal electronic health records (that is, those not linked to clinician held records or embedded in a wider care package) may promote unhealthy consumerism rather than empowerment. ${ }^{21}$ Microsoft and Google each recently launched a personal electronic health record (HealthVault and Google Health, respectively), raising questions about how people's use of such records might occur independently of a professionally led clinical care package.

HealthSpace, an internet accessible personal electronic health record, was introduced in the English National Health Service in 2007, inspired by the Kaiser model. ${ }^{22}$ It is an evolving technology; different functions came on stream at different times, and further changes are planned (box 1). Using a basic HealthSpace account, people may enter values (such as their blood pressure readings) and record healthcare appointments on a calendar. Through an advanced account they can gain secure access to their summary care record (a nationally stored summary of their medical details drawn from the general practitioner record $^{23}$ ), book outpatient appointments, and exchange email style electronic messages with their clinician using a secure link called Communicator. Figure 2 shows the main screen of HealthSpace. Modifications to the HealthSpace user interface were made over the study period but the underlying design and functionality were essentially unchanged.

We have previously reported a survey of NHS patients' attitudes to the clinician controlled summary care record (more than two thirds definitely wanted such a record) and to HealthSpace (fewer than one in four considered trying it). ${ }^{24} \mathrm{We}$ assessed the extent to which HealthSpace had been adopted and used in the three years after its introduction in 2007, the extent to which the benefits anticipated by policy makers had been realised, explanations for non-adoption and abandonment of HealthSpace, and lessons learnt from the introduction and use of personal electronic health records more generally.

\section{METHODS}

\section{Management and governance}

This study, part of the independent evaluation of the summary care record and HealthSpace programmes commissioned by the Department of Health, is reported in detail elsewhere. ${ }^{25}$ The evaluation was overseen by an external steering group chaired by a layperson, with representation from key stakeholders, including patients, professional bodies, academic institutions, and Connecting for Health, which at the time of the study was the "arm's length" agency of the Department of Health responsible for implementing the National Programme for IT. Potential participants were initially approached by someone not directly involved in the research.

\section{Theoretical and methodological approach}

We undertook a mixed method, multilevel case study covering the policy making process, implementation by NHS organisations, and experiences of patients and carers. We followed published guidance for the evaluation of programmes ${ }^{26}$ and interpretive field studies of complex technologies. ${ }^{27}$ We refined these generic approaches into a more specific theory driven methodology, described in detail elsewhere. ${ }^{28}$ In short, we combined qualitative and quantitative approaches to build a rich picture of the case (HealthSpace), making five key assumptions: electronic records do not merely store information but actively shape and constrain care; people and technologies are linked in complex and dynamic networks, hence it is more useful to study the process of socio-technical change than the implementation of a technology; both people and technologies in a network "act," but not in the same way (for example, people have values and feelings whereas technologies do not); everyone's illness has unique features, hence clinical care (including self care) can never be fully standardised; and a technology programme should be studied at both the macro level (for example, national policy, healthcare system, wider social norms, and expectations) and the micro level (for example, particular experiences of patients and professionals).

\section{Data sources and sampling frame}

The table summarises our dataset. As well as reviewing national registration statistics, we studied the adoption and non-adoption of HealthSpace by 56 patients and carers, interviewed 160 staff in national and local organisations, and collected 3000 pages of documents to build a picture of the programme in context. 


\section{Box 1: HealthSpace technology}

As with most software, HealthSpace is an evolving technology. At any time in the evaluation, multiple versions of it existed-including the version that people were actually using, the version that had been approved and was undergoing technical development and limited piloting, and one or more versions that were at the design stage.

A preliminary version of HealthSpace was introduced in 2007, the main features of which were a basic account on to which patients could enter key personal data (for example, weight, blood pressure) and an advanced account through which patients could access their summary care record.

\section{HealthSpace Extension}

By the time we evaluated the preliminary version of the technology in 2008, a business plan for HealthSpace Extension had been submitted. This proposed a more sophisticated product with greater functionality and more extensive links to other parts of the National Programme for IT (for example, booking of appointments, requests for repeat prescriptions, and a secure email link). However, the full functionality of HealthSpace Extension was never developed.

\section{Enhanced version of HealthSpace}

In 2009 an enhanced version of HealthSpace was introduced, which included Communicator, a secure email style messaging connection to the patient's general practice.

\section{Future upgrade}

We were advised that a further upgrade with extended functionality (still to be finalised at the time of writing) was planned for late 2010. We understand this is likely to include a facility to order repeat prescriptions online and a fully online registration process.

\section{Adoption statistics}

Adoption of HealthSpace should ideally be considered in relation to whether people were aware of its existence and whether they had a summary care record, since the advanced HealthSpace account is designed to access the summary care record. Using aggregated data collated nationally by Connecting for Health, we monitored the number of personal letters sent to people with summary care records inviting them to create an advanced HealthSpace account and the number of basic and advanced accounts created nationally. It was not possible to monitor how many people were reached by communications about HealthSpace through national and local mass media (for example, newspapers, radio, public access website), ${ }^{29}$ nor how the national data were broken down by personal factors such as age or sex.

\section{The policy context}

We analysed policies, strategies, and business plans for HealthSpace at two levels. Firstly, we considered their content in a conventional way by assessing the ideas and facts presented. Secondly, we drew on the principles of critical discourse analysis to consider the language, format, and focus of the document, with a view to exploring underlying assumptions and ideologies (see guiding questions in box 2). ${ }^{30}$

\section{Adoption and use of HealthSpace}

The study design and sampling were significantly influenced by the low response rate to our original recruitment strategy. Indeed, the study raised important methodological challenges about how to study nonadoption of an innovation-a task that is essential for overcoming pro-innovation bias in the literature but for which there is no established methodology and few examples from previous research. ${ }^{31}$ We sought to recruit people seeking to use HealthSpace through "front office" registration centres provided by primary care trusts, but because no one agreed to participate (mainly because front office registrations were orders of magnitude lower than expected) we expanded recruitment to general practices, patient organisations, organisations dealing with ethnic communities, and a local development scheme for health trainers (in which lay people are trained to support others in their community to make positive choices about lifestyle). Thus, although we had originally planned to study people who had spontaneously signed up for HealthSpace, our final sample comprised people who consented to try this technology as part of a research project.

We sought to observe participants using HealthSpace in a setting where they felt most comfortable. Many chose to be seen in their homes (table). We observed them registering for a basic HealthSpace account and viewing health data on it. When consent was given, we audiotaped interviews and the participant's efforts to use the technology and used screen capture software to gain multimedia data. We also asked the participants to talk us through their actions in real time.

\section{Studying the design-reality gap in HealthSpace}

An early finding that participants appeared frustrated with HealthSpace prompted us to explore the gap between design and reality-that is, the difference between the assumptions built into the technology and how people actually managed their health and illnesses. To that end, and because of low recruitment of HealthSpace users, we extended our design and sampling frame to include a detailed ethnographic study of people who were not using HealthSpace. We chose people with diabetes as this condition had been flagged in strategy documents as particularly suited to self care via HealthSpace. We began by recruiting from a local diabetes group and a clinical diabetes service and used snowballing (asking participants if they knew someone else with the condition) to extend our sample. We aimed for maximum variety in age, sex, ethnicity, health literacy, IT literacy, stage and severity of condition, presence of other illnesses, and extent of family support.

Drawing on the principle that technology design is usefully informed by a close analysis of the use for which it is intended, ${ }^{32}$ one of us (SH) undertook between one and three periods of detailed ethnographic observation (2-5 hours each) on each of 20 people with diabetes (12 of whom also had other long term conditions). The researcher shadowed them as they went about their daily life, noting how their conditions influenced their activity, what information and communication needs the researcher identified, whether the participant identified these as such, and how they dealt with them. 
Box 2: Examples of questions asked in discourse analysis of policy and strategic documents for HealthSpace (adapted from Blommaert ${ }^{30}$ )

Questions used to guide a detailed critical analysis of policy documents, national strategy documents, and the national business case were:

- Where is the text historically located and in what context was it written?

- What is the text being used to accomplish?

- Who is the author and what is his or her relationship with the text?

- Who are the active agents referred to in the text?

- What are the author's points of departure-that is, what does he or she assume as taken for granted?

- What rhetorical arguments are proposed-for example, what options are presented and in what way is one option depicted as better than others?

- What experts are invoked, and where does their expertise come from?

- What literary devices, such as metaphors, are used?

We invited all participants in this subsample to try using HealthSpace. In the three who agreed, we sought to observe directly how HealthSpace supported or could have supported their efforts to self manage their diabetes and any other conditions. In the 17 who did not want to try HealthSpace, we sought to identify and explore mismatches between its design features (or the assumptions that the participant had made about these) and the lived reality of "self management." We noted other people, such as relatives, friends, peers, the general practitioner, and specialist nurses, who helped the participant manage their conditions and also noted the technologies (such as blood glucose meter, insulin pump, telephone, and internet) that they or their carers used alongside or, more usually, instead of HealthSpace.

The participant was asked to produce a drawing of "how diabetes affects my life." We used this to help map the socio-technical network of people and technologies that they drew upon in managing their condition. We made brief contemporaneous notes while shadowing and immediately afterwards annotated these and added our own reflections, a task that sometimes took several hours. Comments by other members of the research team on these field notes informed subsequent periods of observation and questions to the participant.

Studying adoption and use of email-style messaging Communicator (the secure message exchange function of HealthSpace) was piloted in three general practices. Patients with a range of acute and chronic conditions were invited to try out the technology both through advertising, such as on the practice website and notice board, and opportunistically (by their general practitioners during consultations). Practice staff provided help with registering for an advanced HealthSpace account if needed and asked the participant if they were willing to be interviewed by our team. We recruited 15 participants in this substudy and arranged a face to face interview in the participant's home or, for those who preferred it, a telephone interview. We assured them that responses would be treated confidentially, and anonymised findings fed back to their general practitioner and the national HealthSpace implementation team. We began with a list of semistructured questions (box 3) but encouraged an unstructured narrative if this was forthcoming. In consenting participants, we watched them sending and opening messages.

\section{Analysis of empirical data}

Our dataset comprised quantitative statistics on uptake (which were not amenable to further analysis) plus large amounts of qualitative data of different types, such as field notes, transcripts of interviews, emails, and letters. We read and annotated free text data, developed provisional analytical categories, and iteratively refined these in team discussions using the constant comparative method - that is, comparing each new item of data with an emerging picture of the case as a whole. ${ }^{33}$ We used narrative as a sense making and synthesis tool to weave together the quantitative and qualitative findings into a rich picture of HealthSpace, which depicted key events and upstream causes while also conveying ambiguities and uncertainties. ${ }^{34} \mathrm{We}$ sent drafts of our interpretation to those patients and carers (three of 56) who were interested in seeing them and presented draft findings to participating primary care trusts and national policy makers.

\section{RESULTS}

Adoption rates for basic and advanced HealthSpace

As of end October 2010, 172950 people in England had logged on to the HealthSpace website and entered personal details to create a basic account. These figures were impossible to interpret further as the proportion of the population who knew about HealthSpace at the time, and the proportion of people who actually entered any data or used their basic account, are unknown.

More robust data were available on advanced accounts. As of the end of October 2010, 2442215 people in England, all of whom had had (or were about to have) a summary care record created for them, had received a letter inviting them to open an advanced HealthSpace account. Of these, 11953 $(0.49 \%)$ had completed the first step to opening such an account (that is, logged on to the Connecting for Health website and downloaded a form); 3933 $(0.16 \%$ of those invited) had taken the form to a front office to have it processed and $2913(0.13 \%)$ had activated their advanced HealthSpace account.

\section{Policy and strategy}

HealthSpace was originally conceptualised as part of the National Programme for IT, an ambitious, centrally led programme (estimated cost $£ 12.4 \mathrm{bn}$; $€ 14.3 \mathrm{bn} ; \$ 19.9 \mathrm{bn})$ that aimed to improve informatics support, encourage standardisation, and centralise procurement of information systems in the NHS while at the same time redesigning care systems around the patient. ${ }^{35}$ Policy documents published in 2005-8 were written in a hopeful, upbeat style and 


\section{Box 3: Prompt sheet for exploring how people used HealthSpace or Communicator}

The prompt sheet was adapted flexibly depending on points raised by the interviewee.

- Personal details, such as background, age, sex, ethnicity, work status

- How does the participant rate their own health literacy or knowledge of health issues?

- How does the participant rate their own IT literacy? How much use do they make of computers? (Explore use of IT at home or at work, such as internet banking, shopping, communicating)

- How aware are they of health information sites and do they use these? Has this changed since starting to use HealthSpace or Communicator?

- If willing to discuss, what is the nature of their medical condition and frequency of attendance at the general practice, clinics, etc?

- How and how often did they communicate with their doctor or general practice before using Communicator, and how has this changed?

- How was HealthSpace or Communicator introduced to them and how were its potential benefits and drawbacks described to them?

- Registration process: how did the participant register for a basic or advanced account? Helped by whom? How easy was registration and what issues emerged at this stage?

- What was the participant's expectations of HealthSpace or Communicator before using it?

- What has been their actual experience of using the technology? Prompt for examples of actual usage, especially communications from person to doctor and vice versa

- What type of patient does this participant think would benefit most or least from using this technology?

- What would they change?

- How would they feel if this service was withdrawn?

anticipated five main benefits of HealthSpace: personalisation of care, patient empowerment, reduced NHS costs, patient driven improvements in data quality, and improved health literacy — that is, the availability of HealthSpace would improve people's ability to understand and manage their health) ${ }^{35-39}$ :

HealthSpace is an excellent addition to the SCR [Summary Care Record] programme and has huge potential to improve the safety and efficiency of care by allowing patients to check the accuracy of their SCR and to access detailed information about their own health. In order to take fuller advantage of HealthSpace, we recommend that Connecting for Health trial the use of HealthSpace for patients, particularly those with long-term conditions, to record their own measurements of key health information

House of Commons Health Committee report on the electronic patient record 2007, page $43^{39}$

A triple package, delivered centrally and comprising HealthSpace, the summary care record, and NHS Choices (a website of health information and details of how to access services, www.nhs.uk) was presented in strategy documents as the solution to a particular problem (lack of integration across the NHS leading to fragmentation of care, inefficiency, and risk). This package was depicted as necessarily indivisible and linked to efficiency savings:

HealthSpace needs to be developed in a way which enables it to form an element of the portfolio of public online services complementary to the other elements. That portfolio package must provide services to the patient that are personalised, secure and apparently seamless. Without those features, which can only be delivered by all the elements being offered in a joined up manner, the take up of these services will be damaged with the risk of wasted investment

Strategic outline case for HealthSpace extension, page $13^{40}$

Strategy documents portrayed implementation of the HealthSpace programme in overtly technical and managerial terms ("technology development", "procurement", "deployment"). The main active agents in such documents were the Department of Health, Connecting for Health, software suppliers, policy itself ("policy envisages ... .," "policy drivers"), and the HealthSpace technology (which would "deliver benefits"). Somewhat ironically, patients were referred to generically, collectively, and passively, such as "the public" and even "the mass market," who were depicted as the recipients of HealthSpace's benefits. It was anticipated that although patients might have difficulties using HealthSpace these would generally be remedied by technical modifications to the software.

Strategic and business cases for HealthSpace did not mention patients' expectations of personal electronic health records, motivation to use them, or current self care practices. They acknowledged that segmentation of the target population was possible and identified diabetes as a paradigm condition that could test proof of concept for the technology, although there were no plans to adapt the design for specific user groups or use cases.

The main experts identified in strategy documents were software developers and national implementation leads; neither clinical nor patient expertise was mentioned. A lengthy risk assessment focused exclusively on technical and procedural risks (such as "inadequate specification of requirement by NHS," "information governance and data security requirements [may] change," and even that uptake of HealthSpace accounts would outstrip capacity to process applications $\left.{ }^{40}\right)$. Risks linked to people's feelings, motivation, and values (for example, lack of interest or a perception by patients that the software was not helpful) were not listed.

Early policy enthusiasm for HealthSpace seemed to wane after experiences in 2007-8. The business case submitted in 2008 for introducing extended functionality (see box 1) was not approved by the Department of Health because, according to senior policy makers interviewed for this study, it was considered too high risk, although we were not told why. A business case for a scaled-down version of the programme (not supplied to us but which we understand cost $£ 18 \mathrm{~m}$ compared with an original $£ 98 \mathrm{~m}$ ) was given one year's approval from January 2010 from the "Darzi" funding stream, which lay outside the National Programme for 


\section{Summary of data sources for evaluation of HealthSpace}

Research focus

Adoption rate of HealthSpace

User experience of HealthSpace registration process and early use

Exploratory study of HealthSpace's design-reality gap for people with chronic illness

Use of email-style messaging ("Communicator") by patients and carers

Experience of Communicator by general practice staff

Policy background and perspective of Connecting for Health authorities and primary care trusts

Perspective of citizens and public

\section{Data sources}

National statistics, produced by participating primary care trusts and collated by Connecting for Health, updated weekly, on number of people who had been sent letters about HealthSpace and number of basic and advanced HealthSpace accounts created

Interviews with 21 people who had registered for a HealthSpace account or expressed interest in doing so; observation of them accessing their account and using or trying to use it to manage their illness and risk factors. Nine were interviewed in their homes, seven in a community centre, three in their general practice surgery, and two at their workplace

\section{Interviews with and ethnographic observation of 20 people with} diabetes (12 of whom also had other illnesses), recruited from a forum for patients with diabetes, hospital clinics, a community group, and snowballing from this sample. We shadowed the participant for two or three periods of 2-5 hours each at home and work, noting information needs as they arose and how these were tackled by the participant. Three of these 20 agreed to try HealthSpace as part of the research

Interviews with 13 patients and two carers who had been invited by their general practitioner to participate in the Communicator pilot (eight face to face and seven by telephone at the participant's request). In face to face interviews we observed use of (or attempts to use) Communicator Six interviews and 10 informal meetings or email exchanges with clinicians, managers, and reception staff at three participating general practices

Policy documents, strategy and business cases, committee papers, internal reports, external communications. Interviews and meetings with Connecting for Health staff. Observation notes from boards, committees, and conferences

Interviews and meetings with senior executives, project managers, and IT managers. Observation notes and papers from meetings about summary care record and HealthSpace

Interviews with representatives and field notes from meetings of patient organisations that were considering the implications of HealthSpace. Press articles. Material produced by groups campaigning for civil liberties

IT and was oriented to supporting self management of long term conditions. ${ }^{2}$

\section{Implementation of HealthSpace by NHS organisations}

HealthSpace was initially introduced in two localities, which were early adopters of the summary care record. Implementation work for both these technologies in NHS organisations was complex and labour intensive and it competed for resources and staff time with other policy must dos. ${ }^{2329}$ Key tasks for local teams included delivering a public information campaign, training staff, and running a front office to process patients' applications for advanced HealthSpace accounts.

Project leads from participating NHS organisations repeatedly raised concerns with Connecting for Health in monthly management meetings about the low uptake of advanced HealthSpace accounts, since the benefits predicted (such as lower NHS costs and patient driven improvements to data quality) could not possibly be achieved unless the technology was used. The original strategy had assumed in 2007 that "significantly more than 5\%" of the over- 16 population (page 8) ${ }^{40}$ and in 2008 that "approximately 10\%" of this population (page 13$)^{22}$ would open and use advanced HealthSpace accounts.

Low uptake of HealthSpace seemed to be partly because of patients' limited interest ${ }^{24}$ and partly because the registration process was cumbersome and bureaucratic:
They need to come in to, like the front office, because we check all their identification documents that they need. I have to sign to say that I've seen the three proofs of ID. One's got to be a photograph, like a passport. One's got to be, to have a proof of address so that can be a driving licence or a utility bill, something like that. And then they've got to have a third one, which is definitely like a utility bill that's current; it's got to have been issued in the last 3 months [ . . ]. When I've okayed it, they sign the form and then I give themit's like a little credit card but it's got codes on - and when I've sent the paperwork off to the [national] back office, they then write to the patient and confirm that they've now got their advanced registration and send them a code that they work out with this card, and that gives them access to their record then

Administrator, primary care trust HealthSpace front office

We were informed by Connecting for Health that a future upgrade to HealthSpace will allow online registration.

\section{Efforts by patients to use basic HealthSpace}

Few people who registered for a basic HealthSpace account were willing to be interviewed. The 21 people in this substudy who tried using HealthSpace found it of limited value. None entered any health data on to it and none intended to continue using it in its present form, although some anticipated that a future upgraded version might be more worth while. One reason for abandonment was poor ease of use (for example, what was described as a clunky and counter intuitive access page), which we do not report in detail here as subsequent upgrades improved these features. But the main concern of participants was the fundamental design of the HealthSpace technology and the mismatch between this and their expectations. Despite background literature and explanations by researchers making clear to potential users that they would need to enter their health data themselves, all 21 people interviewed were disappointed and some were angry that they found an "empty" record. Seven of the 21 expected to see their entire general practice held medical record and two expected to see hospital records as well.

Participants in this substudy who had a chronic illness did not see basic HealthSpace as adding value in managing their condition. Some pointed out that self monitoring of health data involves a complex interaction between patient and clinician and that the process of entering and accessing data cannot be meaningfully separated from the wider care relationship:

I don't know why you would document it here, because there's no one else looking at it, there's only you looking at it. [...] You could ask your nurse and then put it in your target. 'What's a good level, what's a good cholesterol to have?' and then put it in a target. I don't know whether people would use it Participant who tried basic HealthSpace once 


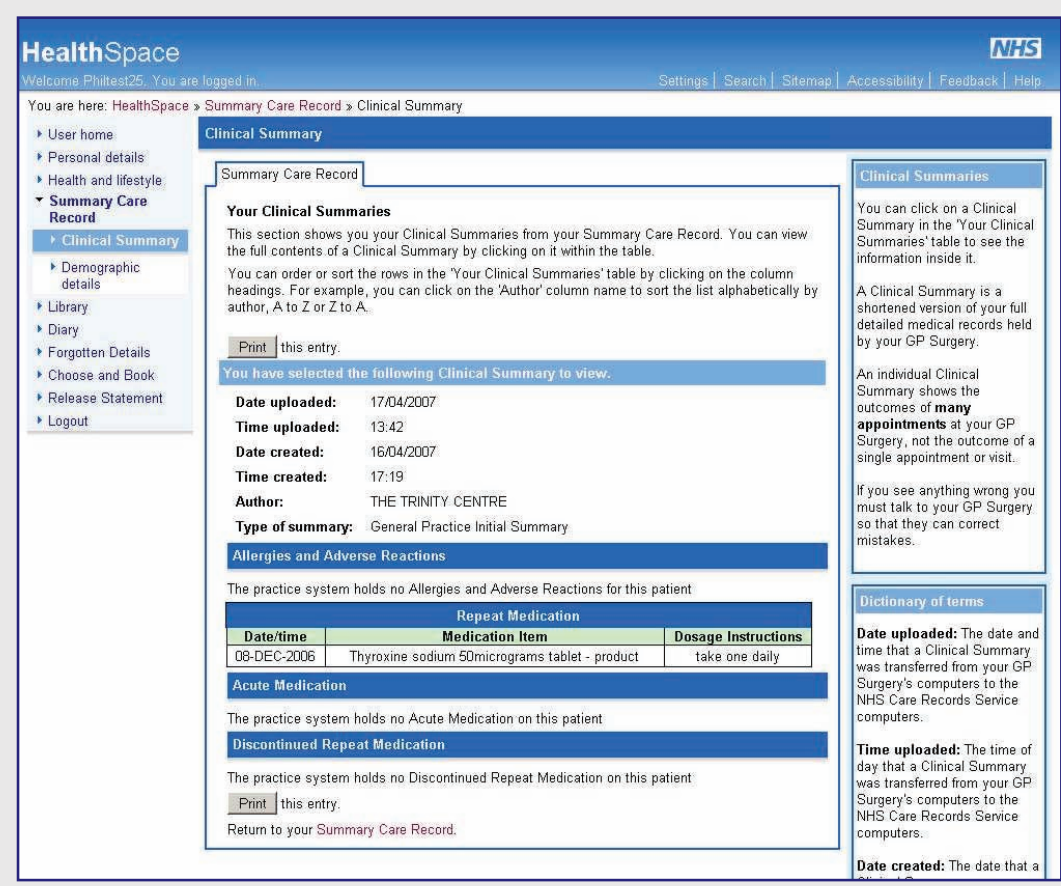

\begin{tabular}{l|l}
\hline Fig 2 & Access screen for HealthSpace, showing link to summary care record
\end{tabular}

We were unable to recruit anyone who had used their advanced HealthSpace account to access their summary care record. This is perhaps unsurprising given that only around one person in 1000 registered for an advanced account and that an unknown proportion of those actually used it. One informant described the "sleeping gym membership" phenomenon: registering for HealthSpace, accessing it once, then losing interest. Nobody interviewed about their use of HealthSpace thought it had changed their use of NHS services.

\section{The design-reality gap}

The 20 participants in this substudy ranged in age from 11 to 69 . All had diabetes and 12 also had other conditions (hypertension, heart disease, kidney disease, thyroid disease, chronic lung disease, arthritis, chronic disability from stroke, visual impairment). Eleven had left school at the minimum school leaving age, 13 were female, and two were from minority ethnic groups. Three of the 20 agreed to try HealthSpace, all of whom abandoned it soon after first accessing it and declared that they were not interested in using it again. Others either were not motivated to reflect on the progress of their condition or thought that this was a task for their doctor or nurse:

What would I do with that information [data entered on HealthSpace] then? It's there for somebody else to analyse but I wouldn't bother with it

Participant with diabetes who chose not to try HealthSpace

Some people in this subsample had no access to computers or the internet at home. More commonly, participants saw these technologies as serving other purposes in their lives (games, shopping, social networking). Some were already using or exploring other ways of documenting and monitoring their condition, such as paper (especially the widely used blood glucose diaries supplied free by manufacturers of monitoring devices) or bespoke software (for example, Excel spreadsheet). One participant who had tried HealthSpace, later became interested in downloads for a digital personal organiser (iPhone apps) and found these more fit for purpose than HealthSpace. This participant later emailed us (unsolicited) a structured table of blood glucose readings over time, generated using a digital application costing around $£ 5$, and commented that this simple task would not have been possible using HealthSpace.

Many participants' information and communication needs were not primarily for codified data (for example, blood glucose levels) but for practical knowledge of how to live with their condition and for emotional support. They tended to get this from other people, such as relatives, local diabetes support group, Facebook, and a nurse led telephone helpline. One participant showed us an online self help group hosted by a charity for patients with diabetes, in which patients and carers exchanged informal stories and tips on topics such as insulin dosing, handling small children with diabetes, and negotiating with health professionals to get the tests and interventions recommended in guidelines.

Our ethnographic observations of participants' efforts to self manage showed that these were sometimes constrained by poverty, an adverse physical environment (for example, poor housing, overcrowding), major family stress, or serious disabilities related or unrelated to their condition, such as depression or stroke. Monitoring and managing long term conditions competed with these other problems for emotional and material resources and was rarely top of the priority list. In this context, HealthSpace with its complex registration procedure, password controlled access, and self completion data fields was described as "faffy" or (more commonly) not considered at all.

\section{The message exchange function}

Despite personal invitations, letters, posters, and "what's new" messages on practice websites, fewer than 100 patients (of a combined list size of around 30000 ) in the three participating practices expressed an interest in using the Communicator messaging function over a six month period. They were given the paperwork to send off to open an advanced HealthSpace account. It is not known how many completed this as the general practitioner is not notified when a patient opens an account. Twenty people who had opened advanced accounts agreed for their details to be passed to us, and of these, 13 wanted to participate in the study. In addition, we interviewed two carers who helped people use Communicator (or used it on their behalf). Age of the 15 participants in this substudy ranged from 25 to 77 (median 48); 10 were female; three 
were from non-white ethnic groups and two from mainland Europe. Most rated their own IT literacy as medium or high and almost all attributed their relevant skills to IT training received at work.

This small and select group of users described the advanced HealthSpace registration process as "pretty straightforward" and the use of Communicator as "easy if you can use email," though two had had their applications turned down by the national back office for reasons that were unclear. Those we observed appeared comfortable and skilled in using the technology. Two carers said that the patient did not have the skills to register or use the technology themselves, and another participant (visually impaired) needed a partner's help because the grid card was not available in large print.

Users of Communicator saw this technology as offering continuity of care with a general practitioner who was popular (hence hard to get an appointment with) and who only worked part time. One thought that Communicator had given them "a full time personal GP all to myself." For many, emotional continuity with "their" general practitioner was more significant than transfer of particular items of knowledge or advice:

My hair started to fall out, and I went out and got a wig.

Dr N replied it's a good idea to get a wig. I'm not doing

a long rigmarole, and it's not that I've got a problem,

I'm just letting her know how things are going. It's nice

that she knows about the wig

Communicator user with a recent diagnosis of cancer (CP12)

Some participants considered Communicator a safety measure for use in emergencies, despite having apparently been instructed not to use it in this way:

In an emergency, I would email.

[Researcher suggests this is inadvisable as doctor only works two days a week in surgery]

In an emergency it would only work during those two days, but on other days we'd have to use the normal method, ringing up

Communicator user (CP05)

Contrary to expectations that Communicator would increase patients' autonomy, some seemed to use it as a means of becoming more dependent on their general practitioner, seeking instructions or affirmation when they might otherwise have made their own decision:

I was quite impressed, I think it's wonderful to be able to type a message to my doctor and he can answer me:

'Do X Y or Z'. I suppose I have blind faith in my doctor. I've never had the inclination into looking up the details of what my medication consists of. [...] I ask doctor if it's safe to take with the other medicines Communicator user (CP04)

Some participants considered that their general practitioner "didn't mind" receiving messages from them; they sent several a week and one checked their account several times daily to see if the doctor had replied. Some thought that emailing their general practitioner directly enabled them to bypass a busy appointment system or negotiate treatment that would otherwise be against the rules (such as asking to attend for flu vaccination outside the flu clinic). Others felt uncomfortable using Communicator, used it rarely, and were concerned that they might offend the doctor (for example, by using the wrong form of address) or intrude on his or her private time:

I know myself how emails encroach on your time, my gut reaction is how is the doctor going to cope with this workload? [ . . ] To be honest, I fear for the other doctors in the practice if this was foisted on them!

Partner of communicator user, who would "never" use it himself

All three general practitioners who participated in the Communicator pilot were initially enthusiasts for the technology; two were employed part time by Connecting for Health and sat on the national steering committee for HealthSpace. Perhaps because of low volumes they did not allocate specific time slots for answering emails from patients nor did they introduce formal systems in the practice to support Communicator use. All had limited success in persuading their partners or other general practitioners to try Communicator and thought that although some patients had undoubtedly benefited from "improved access," uptake overall had been lower than anticipated.

\section{DISCUSSION}

This multilevel case study has shown that the HealthSpace personal electronic health record was poorly taken up by people in England, most of whom perceived it as neither useful nor easy to use. They were disappointed with the amount and type of data available, the need to enter data themselves, and the limited options for sharing these data with their clinician. Enthusiasm from patients and clinicians for HealthSpace's secure messaging function was low, although a few patients who appeared to have high IT literacy found it both useful and easy to use. Policy makers' hopes that HealthSpace would lead to personalised care, lower NHS costs, better data quality, improved health literacy, and greater empowerment were not realised, not least because the technology was not adopted to a major extent and our findings raised the possibility that email-style messaging might reduce autonomy in some patients.

These findings must be interpreted cautiously given the limitations of our patient sample. Although we made efforts to recruit a larger sample of respondents who had spontaneously chosen to adopt HealthSpace and who used it regularly, our final sample was small and consisted mainly of people who had never used it or only did so because we had asked them. They are thus unlikely to represent "real" HealthSpace users (for example, their attitudes contrast noticeably with those 
of official "patient champions" who had spoken publicly in information campaigns about the benefits of HealthSpace in managing their long term condition), but their reactions may be broadly representative of non-users.

The four main possibilities for the limited success of the HealthSpace programme to date are flawed concept, flawed product design, flawed implementation and embedding, and flawed timing - that is, people are not ready for this type of personal health record. Some support exists for all these possibilities, the individual impact of which may have multiplied to explain the dramatic differences between uptake of HealthSpace in England and that of technologies such as Kaiser's My Health Manager in the United States. ${ }^{18}$

HealthSpace was conceptualised more as a passive container for data than as a technology that could actively shape care. Whereas My Health Manager was closely integrated into the overall Kaiser care package, the basic HealthSpace account was intended from the outset to be "untethered" from clinical records or care practices. ${ }^{21}$ The advanced HealthSpace account offered potential for integration into a wider care model through secure messaging and patient access to the summary care record, but the new forms of clinical interaction and wider organisational routines that advanced HealthSpace both presupposed and required were not well worked out, nor were there formal plans to develop these alongside the technology. In retrospect, the notion of HealthSpace as a "data container" to be "deployed" rather than as a component of a socio-technical network (and hence as an opportunity for socio-technical change) was a serious flaw in the original concept.

The original design work for HealthSpace occurred before our evaluation began. People were apparently asked what features they would like to see, but this fell short of the detailed and iterative combination of ethnography, design and redesign, sometimes called technomethodology, which is recommended to produce a definitive user centred technology. ${ }^{41-43}$ Our ethnographic findings, although preliminary and limited to people with diabetes, showed that HealthSpace's underlying design seems to align poorly with many people's self management practices, information needs, and preferred styles of communication. Users wanted more types of data, more functions, and more flexible ways of sharing the data and linking with health professionals. In contrast, My Health Manager offers access to a much wider range of data (but does not require the patient to enter these) and the Kaiser package offers a range of ways of communicating with clinicians. Compared with private sector or open source products, HealthSpace also has limited potential for agile redesign in response to user feedback, since content and functionality is controlled by the state and software upgrades occur slowly and infrequently.

In terms of implementation and embedding, policy makers seem to have treated HealthSpace as a "log on and play" technology and assumed that intended users would need no persuasion to try it and no specific training, support, or incentives to use it. But since UK healthcare is free at the point of delivery, patients may have thought they had nothing to gain by taking on disease management activities currently done by health professionals. The organisation and payment structure of general practice in the United Kingdom does not specifically incentivise email consultations, whereas physicians working for Kaiser are paid per encounter whether this occurs through email or face to face. It is significant that many general practice record systems in the United Kingdom already include functionality for secure email communication with patients, but few practices have enabled this and even fewer use it routinely because of concerns about workload, social boundaries, and the risk of increasing inequalities of access. ${ }^{4-46}$ In contrast, it is established practice for patients in the United States to initiate contact with their physician by telephone or email.

Finally, in terms of readiness, interest in using any form of personal health record currently seems to be low in the United Kingdom. ${ }^{247}$ Anecdotally, patients in the United Kingdom rarely seek access to either paper or electronic health records. My Health Manager was introduced in a very different cultural context. Instead of providing a cradle to grave welfare state, the United States expects citizens to understand their own health needs and take out health insurance or actively sign up to a public healthcare system.

The version of HealthSpace evaluated for this study had lower functionality and greater access hurdles than are intended for the next release (box 1). However, we believe that low adoption rates are unlikely to be fixed by upgrades to the software or by changes to the registration process because although HealthSpace as a technology is evolving, HealthSpace as a programme remains largely unchanged (a relatively non-customisable, relatively untethered product that embodies untested assumptions about the practice of self management), a technology-push implementation strategy and a healthcare system whose readiness for the radical new service model depicted in figure 1 is questionable.

The HealthSpace programme seems to have lacked several critical success factors, which begs the question of why these were not identified at the outset, especially since a lengthy official risk assessment was undertaken. One reason appears to be the technical and managerial focus of early strategy documents. Patients were depicted as passive recipients of HealthSpace's benefits rather than as active agents who have opinions, feelings, relationships, and values, which may explain why these human factors were not identified as mission critical to the programme's success. Risk was assessed by a highly formalised process in which quantitative scores were assigned to policy makers' subjective evaluations of different risks and summed to give an overall risk score. This may have lent a spurious objectivity to estimates of success and diverted attention from systematic qualitative methods such as defending the ideas and plans in front of an audience. It is also possible that the original policy link with patient 


\section{WHAT IS ALREADY KNOWN ON THIS TOPIC}

Personal electronic health records, managed by patients and linked in various ways with clinician led care, are being introduced in many health systems

Expectations for such records include patient empowerment, engagement with self management, reduced healthcare costs, and better continuity of care

\section{WHAT THIS STUDY ADDS}

Adoption of a personal electronic health record by patients in England in 2007-10 was low, and benefits expected by policy makers were not realised over the period of the study

The study raised questions about policy decisions, the technology design process, and implementation in a public sector context

centredness, empowerment, and choice was a "technology of legitimation"-that is, a mechanism by which introduction of a centrally stored electronic patient record system was made more politically acceptable. $^{48}$

In conclusion, it is surely unsurprising that patient engagement and motivation, along with the material properties of the technology in use, have all proved pivotal in the HealthSpace programme to date. The findings of this study suggest that unless personal electronic records align closely with people's attitudes, self management practices, identified information needs, and the wider care package (including organisational routines and incentive structures for clinicians), the risk that they will be abandoned or not adopted at all is substantial. As the NHS considers the next question for such records, we suggest that conceptualising them dynamically (as components of a socio-technical network) rather than statically (as containers for data), and applying user centred design principles more explicitly, might improve their chances of adoption and use.

This evaluation would not have been possible without the cooperation of several people and organisations. Patients and staff in the various field sites allowed us access to their work and experiences, gave freely of their time, and engaged with our desire to gain an honest, richly informed account of events. The evaluation benefited from the Summary Care Record Independent Evaluation (SCRIE) External Advisory Group, chaired by lay member Jenni Bowley, which served as a diverse, critical, and questioning audience for our emerging findings. Members (listed in full in our detailed report ${ }^{25}$ ) included representatives from patient groups, academic institutions, professional organisations, and Connecting for Health. The study was part of the Connecting for Health Evaluation Programme coordinated by Richard Lilford's team at the University of Birmingham. Academic colleagues too numerous to list provided feedback and guidance throughout the study. We thank four $B M J$ reviewers (Hamish Fraser, Peter Singleton, Enrico Coiera, and Erwin Klein Woolthuis) who made detailed comments on an earlier draft of the paper and added additional insights and references.

Contributors: TG designed the study, led the development of the theoretical and methodological approach, helped with data collection, led the data analysis, drafted the paper, and is responsible for the integrity of the work. She is the guarantor. KS and TB led the data collection. SH did the fieldwork for the ethnographic study of 20 people with diabetes. JR helped develop the theoretical and methodological approach and provided expertise in evaluation methodology. All authors helped with analysing data and writing and revising the paper. All aspects of the study (including design; collection, analysis and interpretation of data; writing o the report; and decision to publish) were led by the authors. Sponsors, along with other stakeholders, were given an opportunity to check drafts for accuracy but the final decision on whether to amend drafts was made by the authors in consultation with the SCRIE External Advisory Group.
The views expressed in this publication are those of the authors and no necessarily those of our employing institutions, the individual members of our advisory group, the National Institute for Health Research, or the Department of Health.

Competing interests: All authors have completed the Unified Competing Interest form at www.icmje.org/coi_disclosure.pdf (available on request from the corresponding author) and declare that (1) All authors have support from their employing organisations for the submitted work; (2) No authors have had relationships with organisations that might have an interest in the submitted work in the previous 3 years; (3) Their spouses, partners, or children have no financial relationships that may be relevan to the submitted work; and (4) Authors have no non-financial interests that may be relevant to the submitted work.

Ethical approval: This study was approved by Thames Valley multicentre research ethics committee in January 2007 (06/MRE12/81 and subsequent amendments) and North West 8 multicentre research ethics committee in September 2009 (09/H1013/36 and subsequent amendments)

Data sharing: No additional data available.

1 Bodenheimer T, Wagner EH, Grumbach K. Improving primary care fo patients with chronic illness: the chronic care model. Part 1. JAMA 2002;288:1775-9.

2 Darzi A. NHS next stage review: our vision for primary and community care. Stationery Office, 2008.

3 Cayton H. The flat-pack patient? Creating health together. Patient Educ Couns 2006;62:288-90.

4 Dimick $C$. The empowered patient. Preparing for a new patient interaction. J AHIMA 2010;81:26-31.

5 Szolovitz P, Doyle J, Long WJ, Kohane I, Paulker SG. Guardian angel: patient-centered health information systems. Massachussetts Institute of Technology, 1994.

6 Mandl KD, Szolovits P, Kohane IS. Public standards and patients control: how to keep electronic medical records accessible but private. BMJ 2001;322:283-7.

7 Marshall P. Keeping tabs. How personal health records are changing the face of healthcare. Mark Health Serv 2009;29:20-5.

8 Ferguson T. Consumer health informatics. Healthcare Forum 1995; Jan-Feb:28-33.

9 Fetter MS. Personal health records. Issues Ment Health Nurs 2009;30:652-4

10 Wynia M, Dunn K. Dreams and nightmares: practical and ethical issues for patients and physicians using personal health records. J Law Med Ethics 2010;38:64-73.

11 Roberts J. Personal electronic health records: from biomedical research to people's health. Inform Prim Care 2009;17:255-60.

12 Lawrence D. Footing the bill: patient portals, part I. Healthc Inform 2009;26:20,22,36.

13 Gamble KH. Is it registering? Patient portals, part II. Healthc Inform 2009;26:24,26,28.

14 Washington L, Katsh E, Sondheimer N. Dispute resolution. Planning for disputed information in EHRs and PHRs. J AHIMA 2009;80:26-30.

15 Simborg DW. Consumer empowerment versus consumer populism in healthcare IT. J Am Med Inform Assoc 2010;17:370-2.

16 Silvestre A-L, Sue VM, Allen JY. If you build it, will they come? The Kaiser Permanente model of online health care. Health Aff 2009;28:334-44.

17 Chen C, Garrido T, Chock D, Okawa G, Liang L. The Kaiser Permanente electronic health record: transforming and streamlining modalities of care. Health Aff 2009;28:323-33.

18 Zhou YY, Garrido T, Chin HL, Wiesenthal AM, Liang LL. Patient access to an electronic health record with secure messaging: impact on primary care utilization. Am J Manag Care 2007;13:418-24.

19 Weitzman ER, Kaci L, Mandl KD. Acceptability of a personally controlled health record in a community-based setting: implications for policy and design. J Med Internet Res 2009;11:e14.

20 Maloney FL, Wright A. USB-based personal health records: an analysis of features and functionality. Int J Med Inform 2010;79:97-111.

21 Simborg DW. Consumer empowerment versus consumer populism in healthcare IT. J Am Med Inform Assoc 2010;17:370-2.

22 Howlett P, Beale P. Outline business case for HealthSpace extension. NHS Connecting for Health, 2008.

23 Greenhalgh T, Stramer K, Bratan T, Byrne E, Russell J, Potts HWW. Adoption and non-adoption of a shared electronic summary record in England. BM/ 2010;340:c311.

24 Greenhalgh T, Wood GW, Bratan T, Stramer K, Hinder S. Patients' attitudes to the Summary Care Record and HealthSpace: qualitative study. BMJ 2008;336:1290-5.

25 Greenhalgh T, Stramer K, Bratan T, Byrne E, Russell J, Hinder S, et al. The devil's in the detail: final report of the independent evaluation of 
the Summary Care Record and HealthSpace programmes. University College London, 2010.

26 Patton MQ. Utilization-focused evaluation: the new century. 3rd ed. Sage, 1997

27 Klein HK, Myers MD. A set of principles for conducting and evaluating interpretive field studies in information systems. MIS Quarterly 1999;23:67-93.

28 Greenhalgh T, Stones R. Theorising big IT programmes in healthcare: strong structuration theory meets actor-network theory. Soc Sci Med 2010;70:1285-94

29 Bratan T, Stramer K, Greenhalgh T. "Never heard of it"understanding the public's lack of awareness of a new electronic patient record. Health Expect 2010; published online 15 June.

30 Blommaert J. Discourse: a critical introduction. Cambridge University Press, 2005.

31 Greenhalgh T, Robert G, Macfarlane F, Bate P, Kyriakidou O. Diffusion of innovations in service organisations: systematic literature review and recommendations for future research. Milbank $Q$ 2004;82:581-629.

32 Pratt MG, Rockmann KW, Kaufmann JB. Constructing professional identity: the role of work and identity learning cycles in the customization of identity among medical residents. Acad Manage J 2006;49:235-62.

33 Glaser BG, Strauss AL. The constant comparative method of qualitative analysis. In: Glaser BG, Strauss AL, eds. The discovery of grounded theory. Adline, 1967.

34 Van Maanen J. Tales of the field: on writing ethnography. University of Chicago Press, 1988.

35 Department of Health. Better information, better choices, better health: putting information at the centre of health. Stationery Office, 2004.

36 Department of Health. Choosing health: making healthy choices easier. Stationery Office, 2004.
37 Department of Health. Health reform in England: update and next steps. Stationery Office, 2005.

38 Department of Health. Our health, our care, our say: a new direction for community services. Stationery Office, 2006

39 House of Commons Health Committee. The electronic patient record. Sixth report of session 2006-7. Stationery Office, 2007.

40 Howlett $P$. Strategic outline case for HealthSpace extension. Connecting for Health (internal document), 2007.

41 Oudshoorn N, Pinch TE. How users matter: the co-construction of users and technology. MIT Press, 2005.

42 Pratt W, Reddy MC, McDonald DW, Tarczy-Hornoch P, Gennari JH. Incorporating ideas from computer-supported cooperative work. J Biomed Inform 2004;37:128-37.

43 Hartswood M, Procter R, Slack R, Voss A, Buscher M, Rouncefield M, et al. Co-realisation: towards a principled synthesis of ethnomethodology and participatory design. Scandinavian Journal of Information Systems 2002;14:9-30.

44 Goodyear-Smith F, Wearn A, Everts H, Huggard P, Halliwell J. Pandora's electronic box: GPs reflect upon email communication with their patients. Inform Prim Care 2005;13:195-202.

45 Neville RG, Marsden W, McCowan C, Pagliari C, Mullen H, Fannin A. A survey of GP attitudes to and experiences of email consultations. Inform Prim Care 2004;12:201-6.

46 Wong G. Email consultations in health care: set your auto reply to "no." BMJ 2004;329:1046.

47 Wanless $D$. Securing the public health: taking a long term view. Department of Health, 2004.

48 Harrison S, Mort M. Which champions, which people? Public and user involvement in health care as a technology of legitimation. Soc Pol Adm 1998;32:60-70.

Accepted: 2 September 2010 